\title{
Consumo y medio ambiente en el País Valenciano (1980-2000)
}

\section{Ernest Garcia}

Universitat de València. Departament de Sociologia i Antropologia Social ernest.garcia@uv.es

\section{Resumen}

Al final de los años setenta, tras más de una década de intenso desarrollo económico, algunos rasgos del modelo de consumo de masas se habían asentado en la sociedad valenciana: un sistema de comercialización crecientemente dominado por grandes corporaciones que actúan en mercados mundiales y usan masivamente la publicidad; una dieta con mucha proteína animal, con productos importados de países lejanos y con una presencia grande de alimentos y bebidas muy procesados y envasados; movilidad centrada en el coche; preferencia por la vivienda en propiedad, con tendencia a la suburbanización y requerimientos energéticos en aumento; cultura de «usar y tirar», definida por la corta vida de los productos y la gran producción de residuos. Este modelo de consumo es muy homogéneo e indica el estilo de vida de todos los grupos sociales (excepto los muy pobres). Sus rasgos se han acentuado con el paso del tiempo. El artículo recoge algunos datos (1980-2000) que justifican esta afirmación. Datos acerca de la dieta, el transporte, la vivienda y la producción de residuos sólidos urbanos - cuatro aspectos de la vida que son muy significativos desde un punto de vista medioambiental —. La tendencia hacia más insostenibilidad es impulsada por la evolución de las estructuras productivas y las redes comerciales, así como por la difusión de los rasgos culturales de la modernización. A medida que las prácticas y los hábitos de consumo más «modernos» se convierten en modelo a imitar y extienden su influencia a todos los estratos sociales, esta esfera de la vida apunta, en la sociedad valenciana, a una «modernización antiecológica».

Palabras clave: consumo de los hogares, impacto ambiental, sostenibilidad, dieta, movilidad.

\section{Abstract. Consumption and environment in theValencian Country (1980-2000)}

At the end of the 1970s, after more than a decade of intense economic development, some marks of the mass consumption model were engraved in the Valencian society: a commercialisation system increasingly dominated by large corporations which trade in worldwide markets and use massive advertising; an animal-protein based diet, with products imported from far-away countries, with a strong presence of highly processed and packaged food and beverage; mobility based on cars; preference for owned housing, sub-urbanisation tendency, and growing energy use; «use-and-dump» culture defined by short life of products and high production of waste. This consumption model is highly homogenous and indicates the style of living for all social groups (with the exception of the very poor). Its features have accentuated along time. The article gathers some data (1980-2000) which support this statement. These data correspond to diet, transport, housing and waste 
production -four aspects of life which are strongly significant from an environmental point of view. The trend toward a greater environmental unsustainability is triggered by the evolution of productive structures and commercialisation networks, and the spreading of the cultural features of modernisation. As the more "modern" consumption practices and habits become a model to imitate with an influence on all social strata, in the Valencian society this sphere of life points at an «anti-environmental modernisation».

Key words: household consumption, environmental impact, sustainability, diet, mobility.

\section{Sumario}

El contexto: consumo y medio ambiente

Discusión

en el mundo

Los criterios del análisis

Bibliografía y fuentes estadísticas

Consumo y medio ambiente

en el País Valenciano

Este artículo contiene una presentación descriptiva y analítica del consumo para uso personal (o consumo doméstico o de los hogares) en el País Valenciano, en las dos últimas décadas, desde la perspectiva de su impacto ambiental ${ }^{1}$. El consumo para uso personal es sólo una parte del consumo total de energía y materiales que es ecológicamente relevante —el cual depende también, en buena medida, de los procesos productivos y organizacionales (Stern y otros, 1997; OECD, 1997) —, pero es una parte significativa de dicho total. A la sociedad valenciana de finales de los noventa, podía imputársele, aproximadamente, el 8\% del consumo de agua, el 25\% del de energía, el 44\% del de recursos pesqueros, el $22 \%$ del total de emisiones contaminantes o el $86 \%$ de los residuos sólidos urbanos.

Dedicar atención al consumo doméstico tiene especial interés, por otra parte, en el análisis de las respuestas sociales a las cuestiones de sostenibilidad. La gente tiende a considerar que el consumo es el ámbito en el que tiene más posibilidades de actuación y muestra al respecto una actitud favorable (tabla 1$)^{2}$.

1. Este artículo reproduce con ligeros cambios la ponencia presentada en el seminario «Necesidades, consumo y sostenibilidad», dirigido por Joaquim Sempere y celebrado en el Centre de Cultura Contemporània de Barcelona, del 16 al 18 de octubre de 2002. Su contenido se enmarca en un proyecto de investigación más amplio, dedicado al análisis de problemas de sostenibilidad en el País Valenciano, que se llevó a cabo principalmente entre 1996 y 2002 (ver Almenar, Bono y Garcia, 2000, 2001; Almenar y otros, 2002). Aquí, a efectos de completar la información, se han actualizado algunos datos; sin embargo, el ámbito temporal del análisis es básicamente el indicado en el título.

2. Las respuestas obtenidas a la misma pregunta en otras encuestas realizadas en la década de los noventa, en ámbitos geográficamente más reducidos o sectorialmente más restringidos, son consistentes con las aquí reproducidas (Cabrejas y Garcia, 1997; Garcia, La Roca y Rodríguez, 2001). 
Tabla 1. Actitudes hacia el medio ambiente.

Pregunta: ¿Podría Vd. indicar cuál de las frases siguientes se acerca más a lo que ha sido su actitud hacia los problemas del medio ambiente en los últimos doce meses?

Población de 18 años o más. País Valenciano, 1997

No creo que el medio ambiente sea un problema tan importante como se dice

La verdad es que he tenido que ocuparme de cosas más urgentes

y no he podido pensar mucho en el medio ambiente

He seguido con interés las noticias sobre problemas del medio ambiente

58,9

He dejado de comprar y usar algún producto porque me he enterado

de que es nocivo para el medio ambiente

He colaborado económicamente o he participado en las actividades

de algún grupo ambientalista o ecologista

$\mathrm{Ns} / \mathrm{Nc}$

3,3

Fuente: Almenar, Bono y Garcia, 2000: 502.

Más favorable, al menos, que hacia el compromiso activo con el movimiento ecologista o hacia la intervención en los procesos de producción. Una minoría significativa de la población valenciana (en torno al 15\%) se muestra básicamente insensible a los problemas del medio ambiente. La actitud mayoritaria (en torno al 60\%) es de interés por el tema: se trata de algo de lo que hay que estar informado, ya que se considera preocupante (y se opina, pues, que debe ser materia de atención por parte de los poderes públicos). Una minoría significativa (en torno al 20\%) considera que también debe ser objeto de la propia actuación en tanto que consumidores o consumidoras. Finalmente, una minoría más exigua (3-4\%) entiende que el medio ambiente ha de incorporarse a su práctica sociopolítica. En principio, pues, el consumo es el ámbito de las prácticas sociales en el que cabría esperar más incidencia de los comportamientos individuales.

Algunas manifestaciones de la situación reflejada en el párrafo anterior comienzan a ser perceptibles: el surgimiento de mercados para productos ambientalmente benignos, la aparición de estilos de vida alternativos, etc. Hasta ahora, sin embargo, sus efectos son marginales (si se juzgan en base a la evolución del consumo en magnitudes agregadas, a la que me referiré a continuación).

\section{El contexto: consumo y medio ambiente en el mundo}

En diferentes sociedades, en distintos lugares del mundo, el proceso de modernización ha tenido como resultado un modelo de consumo que presenta, en general, los rasgos siguientes: una concentración creciente de las estructuras de distribución y comercialización, a menudo con una implantación transnacional y con un fuerte apoyo publicitario; una dieta con mucha proteína ani- 
mal y con una significativa presencia de alimentos y bebidas muy procesados y envasados y transportados a largas distancias; una movilidad basada en el automóvil privado, en el marco de un sistema de transporte organizado al servicio del mismo; una forma de alojamiento caracterizada por el aumento de la superficie construida y por la tendencia a la suburbanización; una cultura de «usar y tirar», marcada por la moda, la poca durabilidad y reparabilidad de los productos y la elevada producción de residuos. El modelo de consumo así resumido es muy homogéneo en los ámbitos sociales más modernizados. Indica el estilo de vida de casi todos los grupos sociales en los países industrializados y de las minorías más o menos amplias de clase media en los países en vías de desarrollo (Durning, 1992; Goodwin, Ackerman y Kiron, 1997; Crocker y Linden, 1997; Rosenblatt, 1999).

Hay una interesante serie de estudios - no limitada a los aspectos medioambientales y con perspectivas no siempre coincidentes - sobre los efectos de la difusión mundial del modelo de consumo al que antes he hecho referencia, así como sobre las complejas interconexiones ecológicas y sociales implicadas por el mismo (Teitel, 1992; Ritzer, 1996; Ryan y Durning, 1997; Matthews y Hammond, 1999; Schlosser, 2001). En cualquier caso, los datos sugieren, plausiblemente, que los estilos de vida más modernos están incrementando la presión sobre los sistemas naturales de soporte de la vida más de lo que correspondería linealmente al crecimiento demográfico; y que, en el periodo más reciente, la contribución relativa a ese efecto de las minorías de clase media de las sociedades en vías de desarrollo es significativa. Dicho de otra manera: parece que, por lo que respecta al consumo, los fenómenos que podrían asociarse más claramente a la hipótesis de una modernización ecológica (los mercados de alimentos orgánicos y otros productos «verdes», las experiencias de sencillez voluntaria más o menos distinguida (Sachs, Loske, Linz y otros, 1998: 125) o la participación creciente de los servicios en el conjunto del gasto) no implican - $\mathrm{O}$, al menos, no lo hacen aún- un cambio en la tendencia de costes ambientales crecientes. En general, los consumos ambientalmente más costosos han venido aumentando a un ritmo relativamente más rápido que el de la capacidad de gasto considerada en su conjunto, tanto en las sociedades industrializadas como en las que están en vías de industrialización.

En las últimas décadas, la expansión económica ha tenido como consecuencia un incremento en la capacidad de gasto para el consumo, tanto en las sociedades industrializadas como, en promedio, en el resto del mundo. El incremento del gasto se ha traducido en un mayor consumo de bienes asociados a un impacto ambiental más elevado (coches y gasolina, carne, papel). Tanto la dinámica posicional de imitación y emulación como las exigencias básicas del desarrollo humano, empujan en grados diferentes en esa dirección (por ejemplo: el consumo de papel tiene que ver con la distinción cultural, pero también con la alfabetización). En 1996, el número de coches por cada mil habitantes había aumentado en el mundo desarrollado en algo más de un 10\% respecto al de 1990; en el conjunto de los países en vías de desarrollo, en un $66 \%$. El consumo de gasolina por persona en 1997 se había incrementado en 
Tabla 2. Consumo de productos significativos por su impacto ambiental.

\begin{tabular}{|c|c|c|c|c|c|c|c|c|}
\hline & \multicolumn{2}{|c|}{$\begin{array}{l}\text { Coches } \\
\text { (por } 1.000 \text { hab.) }\end{array}$} & \multicolumn{2}{|c|}{$\begin{array}{l}\text { Gasolina } \\
\text { (por año, } \\
\text { litros por persona) }\end{array}$} & \multicolumn{2}{|c|}{$\begin{array}{l}\text { Carne } \\
\text { (por año, } \\
\text { kg. por persona) }\end{array}$} & \multicolumn{2}{|c|}{$\begin{array}{l}\text { Papel } \\
\text { (por año, } \\
\text { kg. por persona) }\end{array}$} \\
\hline & 1990 & 1996 & 1987 & 1997 & 1988 & 1998 & 1988 & 1998 \\
\hline $\begin{array}{l}\text { Asia } \\
\text { (sin Oriente Medio) }\end{array}$ & 16 & 23 & 30 & 50 & 15 & 25 & 18,1 & 26 \\
\hline Europa & 224 & 289 & 232 & 303 & 58 & 72 & 80 & 111,5 \\
\hline $\begin{array}{l}\text { Oriente Medio } \\
\text { y Norte de África }\end{array}$ & - & 43 & 127 & 149 & 21 & 22 & 9,9 & 15,5 \\
\hline Africa Subsahariana & 11 & 14 & 31 & 31 & 12 & 13 & 5,3 & 4,8 \\
\hline$\underline{\text { Norteamérica }}$ & 563 & 484 & 1.618 & 1.637 & 113 & 120 & 300,3 & 295 \\
\hline Centroamérica y Caribe & - & 69 & 182 & 204 & 32 & 39 & 31,1 & 34,7 \\
\hline Sudamérica & - & 32 & 122 & 148 & 48 & 61 & 26,5 & 34,9 \\
\hline Oceanía & 344 & 364 & 710 & 692 & 98 & 93 & 126,9 & 133,7 \\
\hline Países desarrollados & 296 & 326 & 548 & 626 & 66 & 77 & 137,9 & 160,2 \\
\hline $\begin{array}{l}\text { Países en vías } \\
\text { de desarrollo }\end{array}$ & 9 & 15 & 39 & 55 & 18 & 26 & 11 & 17,5 \\
\hline
\end{tabular}

Fuente: WRI, 2001.

un $14 \%$ de la cantidad de 1987 en los países desarrollados; en un $41 \%$ en el resto del mundo. El de carne, en un $17 \%$ y un $44 \%$, respectivamente, entre 1988 y 1998 . En un $16 \%$ y un $59 \%$, respectivamente, el de papel (FNUAP, 2001; WRI, 2001). En el periodo más reciente, pues, el consumo de varios de los productos con mayor impacto ambiental ha seguido en aumento en todo el mundo, haciéndolo más rápidamente en aquellas sociedades en las que el punto de partida era más bajo (tabla 2).

\section{Los criterios del análisis}

En términos generales (haciendo abstracción de las técnicas de producción primarias), el impacto ambiental de la dieta tiende a aumentar en función de tres factores. Uno de ellos es la proporción que, en la composición de la misma, representan productos que suponen comer más arriba en la cadena trófica: en general, las dietas con más proteína animal son ambientalmente más costosas que las relativamente más vegetarianas. Más todavía si esa proteína animal procede de carnes rojas (Rifkin, 1992; Tansy y d'Silva, 1999). La producción de una unidad de proteína en carne de bovino requiere quince veces más tierra y más agua que en carne de pollo (Smil, 2001: 159-160). Cada kilogramo de carne de bovino cuesta alrededor de dieciséis kilogramos de suelo erosionado, exige a menudo hasta quince veces más agua que una cantidad equiva- 
lente de proteína vegetal y es el resultado de la conversión de una cantidad más de diez veces superior de ésta última (Rifkin, 1992: 185-203; Lappé, 1982: 76-77; Pimentel y Pimentel, 1979: 58). Otro factor relevante es la distancia recorrida por los alimentos desde el lugar en que son producidos hasta el punto de su consumo final: a medida que esa distancia aumenta, lo hacen también la energía usada en el transporte y los requerimientos de conservación y envasado. Por eso, en general, los alimentos locales son ambientalmente menos costosos (Böge, 1996; Pirog y otros, 2001). En tercer lugar, el impacto ambiental tiende también a aumentar a medida que lo hace el grado de procesamiento previo al consumo final (el consumo de alimentos frescos suele ser ambientalmente más benigno que el de preparados, precocinados, etc.). Los tres factores no siempre se agregan linealmente. Diferencias locales (en el clima, los suelos, etc.) pueden dar lugar a excepciones; por ejemplo: el consumo de tomates en Escandinavia puede ser energéticamente más costoso si se cultivan en invernaderos de Suecia que si se importan de España (Carlsson-Kanyama, 1998). De todos modos, la regla cualitativa que, en materia de dieta, identifica «ecológico» con orgánico, local, fresco y bajo en la cadena trófica suele resultar razonable. Para evaluaciones más precisas, hay diversas técnicas disponibles (Coley y otros, 1998; Carlsson-Kanyama, 1997; Heller y Keoleian, 2000).

Criterios cualitativos similares, que permitan, por lo menos, establecer escalas cuasi ordinales, son aplicables también a otras esferas del consumo. Así, el impacto ambiental del transporte aumenta, en general, a medida que lo hacen la distancia recorrida y la velocidad requerida para llegar al punto de destino. También, cuanto más intensivos en energía y materiales son los medios utilizados (el transporte basado en el coche privado es menos sostenible que el basado en medios colectivos motorizados, y éste último lo es menos que el realizado en bicicleta o a pie) (Sachs, 1992; Freund y Martin, 1993; Durning, 1996; Estevan y Sanz, 1996; Newman y Kenworthy, 1999).

Dada la gran complejidad de la vivienda, hay muchos factores que influyen en la eficiencia ecológica de la forma de residencia. Materiales y procesos de construcción, proximidad o lejanía entre los diseños y las condiciones bioclimáticas, durabilidad o ritmo de sustitución de los elementos duraderos (muebles, equipos...), magnitud de los flujos de energía y materia (agua, electricidad, residuos...), distancia entre el lugar de residencia y los destinos cotidianos, etc. (Harland, 1995; Sachs, Loske, Linz y otros, 1998: 148-157; Brower y Leon, 1999; Camagni, Gibelli y Rigamonti, 2002).

En base a criterios como los arriba esbozados, es posible sintetizar los consumos con mayor impacto ambiental en una lista razonablemente corta: coches, carne, productos agrícolas, iluminación y artefactos eléctricos, calefacción y refrigeración, construcción de la vivienda, agua consumida y aguas residuales... (Brower y Leon, 1999; Lorek y Spangenberg, 2001). De hecho, para comparaciones internacionales, los datos relativos a algunos de esos ítems se suelen tomar como suficientemente significativos (UNDP, 1998; Matthews y Hammond, 1999; FNUAP, 2001; WRI, 2001; OECD, 2002). 
En este artículo, se examinan algunos datos relativos a la alimentación, al transporte y a la vivienda. Se ha completado el análisis considerando la producción de residuos sólidos urbanos, un indicador útil para aproximarse al impacto ambiental agregado del consumo doméstico y, en particular, al alcance de la «cultura de usar y tirar».

\section{Consumo y medio ambiente en el País Valenciano}

Los resultados obtenidos en el análisis del consumo en la sociedad valenciana son consistentes con lo arriba apuntado. Desde que culminó la transición desarrollista, en la segunda mitad de los años setenta del siglo pasado, el modelo antes descrito de consumo de masas se muestra consolidado y, en el marco del mismo, la participación relativa en el gasto de los productos con mayor impacto ambiental parece haber ido en aumento. Antes de entrar en más detalles, registraré los datos correspondientes a algunos consumos que se caracterizan tanto por la significativa participación en ellos de los hogares como por su importante contribución a la presión ejercida sobre el medio ambiente. El número de vehículos de motor, el consumo aparente de cemento, el uso de electricidad y la generación de residuos sólidos urbanos han aumentado sensiblemente más deprisa que la población (gráfica 1). Obtenemos, con ello, una visión preliminar del cuadro que, a continuación, se dibujará más en detalle mediante el repaso de algunos de los principales capítulos del gasto de los hogares.
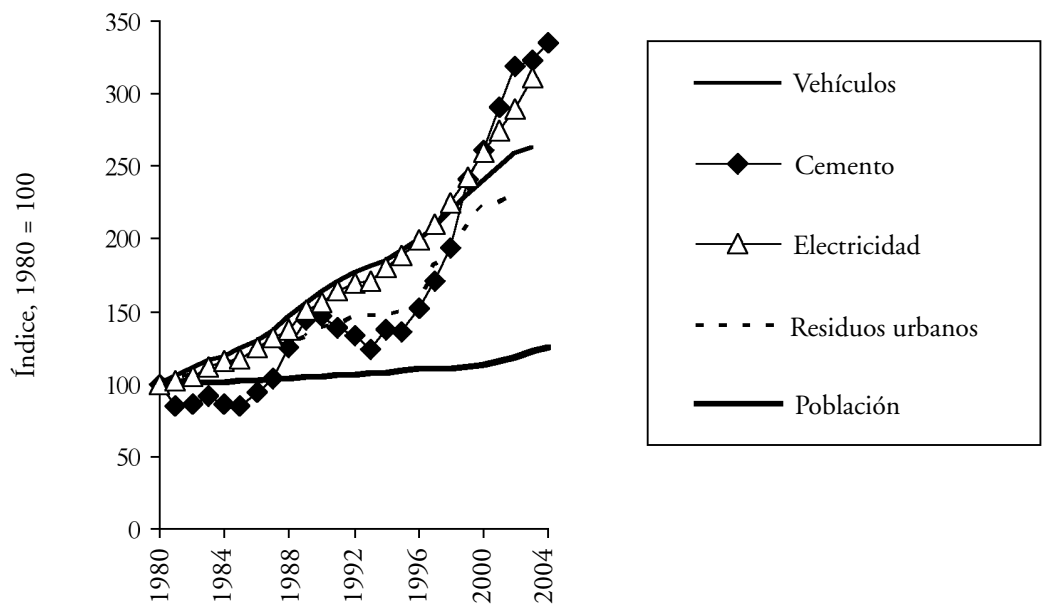

Gráfica 1. Población, consumos y residuos. País Valenciano, 1980-2004.

Fuente: INE, IVE, diversos años. Elaboración propia. 


\section{La dieta}

El análisis de la evolución de la dieta revela un cuadro particularmente complejo. De acuerdo con los datos disponibles, el consumo de alimentos en los hogares valencianos ha descendido en términos reales durante la última década del siglo XX, tanto si se considera la cuestión en moneda constante como en cantidades físicas. El descenso es particularmente acusado en lo que respecta a los productos que requieren elaboración, y más acusado cuanta más elaboración requieren: verduras y legumbres, carne y pescado, incluso frutas. De los grupos de alimentos básicos, sólo los cereales conocen una sorprendente recuperación, parcialmente vinculada al ascenso muy grande de toda clase de productos muy procesados, listos para comer (tabla 3).

El desarrollo, desde los sesenta hasta los noventa, implicó la sustitución de los guisos de olla por la carne y el pescado a la plancha. La globalización parece implicar que incluso pelar un plátano requiere más tiempo del disponible. Todo lo que no pueda consumirse directamente sacado de la nevera o recalentado en un horno rápido, disminuye su presencia en la dieta. Rasgos que se extienden también, seguramente, a muchas de las comidas realizadas fuera del hogar (sobre todo en lugares conectados más o menos directamente con la actividad: comedores de empresa o escolares, etc.), es decir, en lugares donde la cocina y los ritos de consumo responden en buena medida a las mismas reglas. Pese a las resistencias derivadas de la tradición cultural, el resultado combinado de todo ello apunta a la extinción simultánea de la dieta mediterránea, la cocina de la abuela y la comida en familia, como efecto de la escasez de tiempo en la acelerada sociedad contemporánea y de la capacidad de la industria agroalimentaria y de las grandes cadenas de distribución para responder a la demanda fundamental: ¡rápido y barato! El nuevo paradigma de la alimentación junto al Mare Nostrum parece ser la telepizza con Coca-cola light (cada cual en su cuarto, surfeando en Internet). O, en la versión para clases trabajadoras, el bocadillo o la pizza precocinada y ultracongelada adquirida en una gran superficie, ingerida frente al televisor (gráfica 2$)^{3}$.

3. Para el cálculo de la cantidad consumida de productos alimenticios muy procesados, se han incluido las rúbricas de la clasificación de bienes y servicios COICOP/HBS — desagregada a siete dígitos-, que contienen, de forma predominante o significativa, aunque no exclusiva, productos como los siguientes: pastas azucaradas y pasteles industriales (gofres, etc.); pizzas; preparados de cereales; productos de cereales conteniendo carne, pescado o verduras; preparados de carne y platos preparados a base de carne, pescado o verduras; productos de leche fermentada y postres a base de leche; frutas y verduras congeladas y en conserva; productos derivados de tubérculos; confituras; helados; preparados con azúcar; salsas y condimentos; sopas preparadas... Se trata, pues, de una aproximación a la percepción cuantitativa de una tendencia, más que de una medida precisa.

Todos los datos para 1999 se han estimado a partir de las cifras disponibles sobre gasto anual y precios unitarios de la ECPF (base de 1997). Otras fuentes ofrecen resultados diferentes. El informe sobre alimentación del Ministerio de Agricultura, por ejemplo, registra cifras que son algo más altas para el consumo de carne en el hogar y algo más bajas para el de verduras (Ministerio de Agricultura, Pesca y Alimentación, 2000). En cualquier caso, 
Tabla 3. La alimentación en el País Valenciano en 1999. Productos cuyo consumo en el hogar ha aumentado o ha disminuido respecto a 1990-1991.

\begin{tabular}{|c|c|}
\hline Han aumentado & Han disminuido \\
\hline $\begin{array}{l}\text { Pan (no integral e integral) } \\
\text { Otros panadería } \\
\text { Pastas } \\
\text { Preparados a base de cereales } \\
\text { Preparados de carne } \\
\text { Crustáceos y moluscos } \\
\text { Preparados y conservas de pescado } \\
\text { Leche semidescremada y descremada } \\
\text { Yogures } \\
\text { Preparados a base de leche } \\
\text { Frutas congeladas y en conserva } \\
\text { Zumos de frutas envasados } \\
\text { Verduras congeladas } \\
\text { Derivados de tubérculos } \\
\text { Zumos de vegetales } \\
\text { Confitería y helados } \\
\text { Salsas y condimentos } \\
\text { Sopas y otros preparados } \\
\text { Agua mineral } \\
\text { Bebidas gaseosas }\end{array}$ & $\begin{array}{l}\text { Arroz } \\
\text { Carnes rojas } \\
\text { Pollo, gallina y otras aves } \\
\text { Charcutería } \\
\text { Otras carnes y casquería } \\
\text { Merluza y pescadilla fresca } \\
\text { Merluza o pescadilla congelada } \\
\text { Otros pescados } \\
\text { Pescados secos, ahumados... } \\
\text { Leche entera } \\
\text { Leche en polvo, condensada... } \\
\text { Queso y requesón } \\
\text { Huevos } \\
\text { Mantequilla y margarina } \\
\text { Aceite de oliva y otros aceites } \\
\text { Cítricos } \\
\text { Otras frutas } \\
\text { Frutos secos } \\
\text { Hortalizas de hoja y hierbas } \\
\text { Coles } \\
\text { Hortalizas de fruto } \\
\text { Hortalizas de raíz o bulbo } \\
\text { Legumbres secas } \\
\text { Verduras en conserva } \\
\text { Patatas } \\
\text { Azúcar } \\
\text { Chocolate } \\
\text { Café, té e infusiones } \\
\text { Cacao } \\
\text { Vino } \\
\text { Cerveza } \\
\text { Espirituosos y licores }\end{array}$ \\
\hline
\end{tabular}

Fuente: INE, Encuesta de Presupuestos Familiares 1990-1991, Encuesta Continua de Presupuestos Familiares (base 1997) 1999. Elaboración propia.

el patrón de evolución aquí presentado es consistente con las diversas fuentes consultadas y responde a una bien contrastada tendencia de la alimentación en España (Ministerio de Agricultura, Pesca y Alimentación, 2001). Queda pendiente una contrastación más sólida de si, como parece, los rasgos básicos de esa tendencia se presentan en el caso valenciano de una forma más intensa y acelerada que en el conjunto de la sociedad española. 


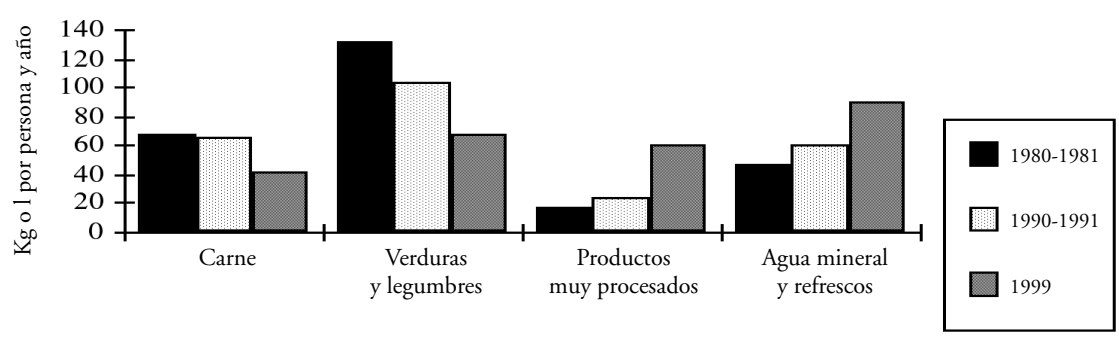

Gráfica 2. Alimentación en el hogar, PV, 1980-1999.

Fuente: INE, 1984, 1994; ECPF, 1999 (soporte magnético). Elaboración propia.

Desde el punto de vista de la sostenibilidad medioambiental, el cuadro es notablemente contradictorio. El consumo en los hogares de los productos que implican mayor presión sobre los sistemas naturales (carnes rojas, pescado) se ha moderado apreciablemente, aunque es probable que tengan una presencia más grande en las comidas fuera de casa ${ }^{4}$. La preocupación por los efectos de la alimentación sobre la salud, la ampliamente difundida convicción de que una dieta con menos carne y grasas es más saludable, puede estar impulsando este efecto (así como el menor desgaste físico en el trabajo y el difundido deseo de guardar la línea). En cambio, es posible que el consumo previo de energía, en procesado y transporte, lo compense sobradamente en términos de costes ambientales, la que refuerza la tendencia de la dieta moderna a depender muy estrechamente de la oferta de combustibles fósiles baratos. El caso de la sustitución del agua obtenida de la red pública por agua embotellada o refrescos gaseosos - a la hora de satisfacer una necesidad tan básica como la sed- es particularmente ilustrativo. Las redes de suministro de agua potable, uno de los rasgos más indiscutibles del progreso en la sociedad industrial, envejecen y ven deteriorada su calidad de forma imparable. Una parte considerable de la población valenciana se ve forzada a un cambio, bien porque el agua que sale del grifo ya no es del todo segura sanitariamente (por exceso de nitratos), bien porque ya no es en absoluto potable (por ser demasiado salobre, como ocurre en diversos municipios del litoral turístico).

4. Varios de los cambios recientes en las costumbres de alimentación, desde los espacios donde se come y se bebe, hasta la compleja composición de ingredientes en muchos productos preparados, implican que los medios que han venido siendo habituales para obtener información (la encuesta de presupuestos familiares, los informes sobre alimentación del Ministerio de Agricultura o los dispositivos similares de otros países) produzcan datos cada vez más opacos desde el punto de vista que me ocupa aquí. Como se ha señalado (Smil, 2001), para propósitos como el de este trabajo, esos datos resultan cada vez más insatisfactorios. 


\section{Transporte y vivienda}

El rasgo más visible en el ámbito de la movilidad es el incremento en los niveles de motorización. En 1997, el 44\% de los desplazamientos de las personas mayores de edad al centro de trabajo o de estudio se hacían en vehículos privados ocupados por una sola persona; el 6\%, en vehículos compartidos por más de una persona, y el 15\%, en medios públicos de transporte. El recurso al vehículo privado es todavía mayor en los desplazamientos de ocio, aunque en este caso es también más frecuente el uso compartido (Garcia, 1999). Una encuesta posterior, ha registrado una utilización aún mayor del vehículo privado ( $57 \%$ de los viajes de más de diez minutos) y menor de los transportes públicos (9\%) (MIFO, 2000). En 1999, había un coche en el 87\% de los hogares valencianos (y más de uno en una cuarta parte de ellos). Aunque no hay datos directos recientes, parece haberse incrementado también la distancia recorrida, como lo sugiere la evolución del consumo de combustibles de automoción (gráfica 3). Aunque los nuevos modelos suelen ser más eficientes y consumen menos combustible por unidad de distancia recorrida, la población ha comprado coches más grandes y potentes y se ha movido más en ellos. De esta manera, el «efecto rebote» ha absorbido sobradamente las eventuales mejoras en la eficiencia.

En el País Valenciano, un rasgo del sistema de habitación que contribuye poderosamente a la presión ambiental es la sobredimensión relativa del parque de viviendas. Según los registros censales, el número de viviendas familiares en el País Valenciano se incrementó en un 20,61\% entre 1981 y 1991, pasando de 1.732.149 a 2.089.063 (algo más de una por cada dos habitantes). En 1991, el número de viviendas principales o residencias habituales era de

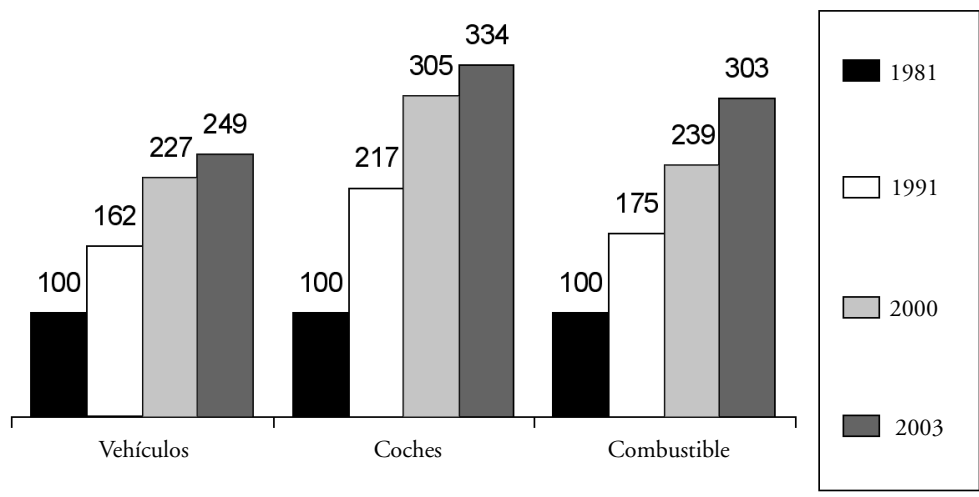

Gráfica 3. Vehículos a motor, automóviles de turismo y consumo de combustible de automoción, PV, 1981-2003. Valores relativos, $1981=100$. 
1.205.331 (un 58\% del total). Había 517.694 viviendas secundarias (una cuarta parte de las existentes) y 313.658 desocupadas (el $15 \%$ del total). En la década siguiente, el incremento en el número de viviendas volvió a ser relativamente muy superior al de la población. El censo de 2001 registró 2.547.775 viviendas, entre las que 1.492 .744 eran principales, 564.086 secundarias y 444.823 estaban vacías. Una característica del parque valenciano de viviendas es, pues, su elevada magnitud respecto a la población residente.

El rasgo comentado depende en parte de la demanda de segundas residencias, por parte tanto de la población residente en la comunidad autóno$\mathrm{ma}$, como de inversores y usuarios foráneos. Es, en todo caso, uno de los aspectos reseñables de una dinámica constructora de intensidad excepcionalmente alta. Entre 1992 y 2000 los visados para edificios residenciales representaron más de 58 millones de $\mathrm{m}^{2}$ a construir, con una presencia notable de la edificación dispersa (el 26,8\% de los visados correspondía a viviendas unifamiliares, aisladas o adosadas) (MIFO, 2001b). De acuerdo con esta misma fuente, de los 50 municipios españoles con mayor número de viviendas visadas en relación con su población en dicho periodo, 14 eran valencianos; y 4, de los 10 primeros (Torrevieja, Guardamar, Rojales y Pilar de la Horadada). En 1997, la superficie del estoc de viviendas se había incrementado en un $13 \%$ respecto a 1990 , pasando de 193,6 a 218,8 millones de $\mathrm{m}^{2}$ (MIFO, 2001a). En 1998, el suelo calificado oficialmente como urbano y urbanizable sumaba 137.446 hectáreas, cerca del 6\% de la superficie total de la comunidad autónoma (IVE, 2002). Todo lo arriba apuntado, sumado a lo que los registros oficiales no recogen o recogen de modo imperfecto, implica una presión dramática sobre el territorio. La forma más clara de describirla es, probablemente, calcular la deposición de cemento por unidad de superficie, que — como puede verse en la gráfica 4 - alcanza valores relativamente muy elevados.

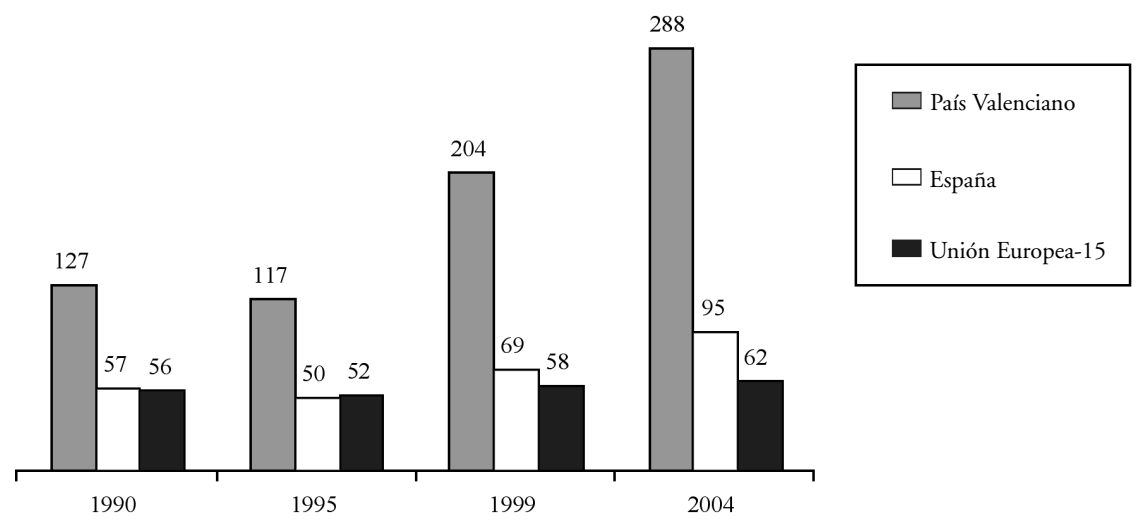

Gráfica 4. Densidad física de la urbanización. Toneladas de cemento por $\mathrm{km}^{2}$ y año. Fuente: INE, Oficemen, Cembureau. Elaboración propia. 


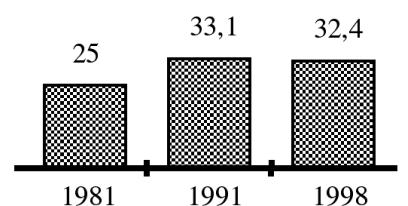

1981

1991

1998

Gráfica 5. Superficie útil habitable de las viviendas principales, metros cuadrados por persona, PV, 1981-1999. Fuente: INE, Encuesta de Presupuestos Familiares. Elaboración propia.
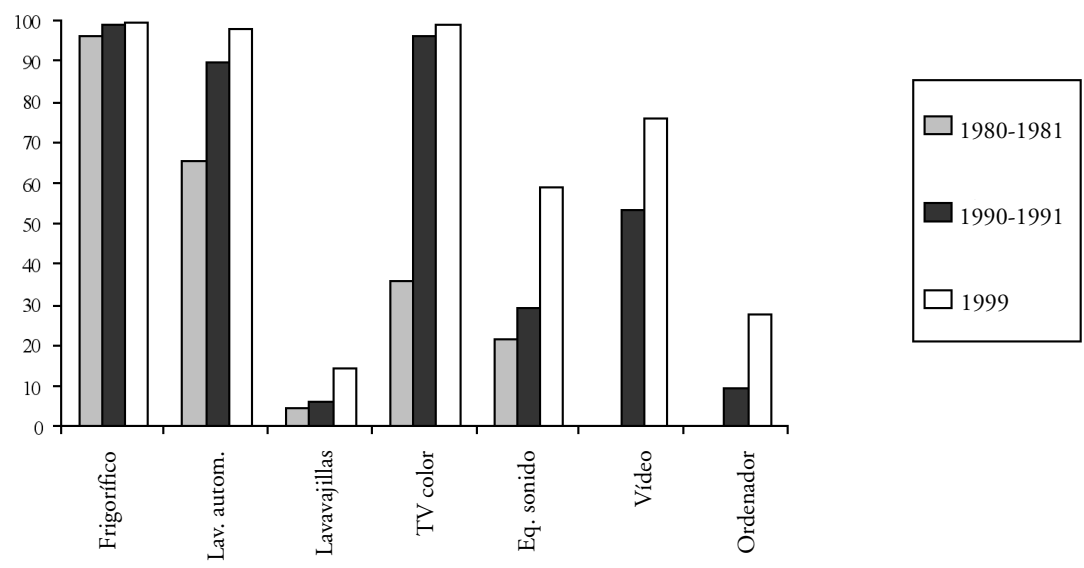

Gráfica 6. Aparatos eléctricos en los hogares, PV, 1980-1999, \%.

La situación reseñada no implica necesariamente que el espacio habitado cotidianamente - la superficie disponible por persona en la vivienda principal- esté aumentando. De hecho, parece no ser así (gráfica 5). Sin embargo, cada vez se instalan sobre esa superficie más aparatos dependientes de la electricidad para su funcionamiento (gráfica 6). Como consecuencia de ello (y también del consumo de combustibles fósiles para climatización), el uso de energía en los hogares ha crecido significativamente (gráfica 7).

5. La serie relativa a superficie de las viviendas (gráfica 5) debe mirarse con cierta precaución. Los datos proceden de la EPF, corresponden a las respuestas al cuestionario de esa encuesta y se refieren a superficie útil habitable. Aunque pueden considerarse como una aproximación internamente consistente, la cuestión podría también abordarse desde otros ángulos. Por ejemplo: la estadística sobre precios del Ministerio de Fomento, referida a superficie construida y estimada a partir del mercado inmobiliario, recoge una cifra de 38 metros cuadrados en 1998 (MIFO, 1999). En cualquier caso, a los efectos de este trabajo, el número creciente de segundas residencias utilizadas o poseídas por la población valenciana implica que la superficie disponible por persona habría seguido aumentando, incluso si no fuese éste el caso en lo referente a las viviendas principales. Por último: si nos limitásemos a dividir la superficie del estoc de viviendas por la población de la comunidad autónoma, entonces habría que elevar la superficie construida, hacia el final del siglo XX, hasta 55 metros cuadrados por persona. 


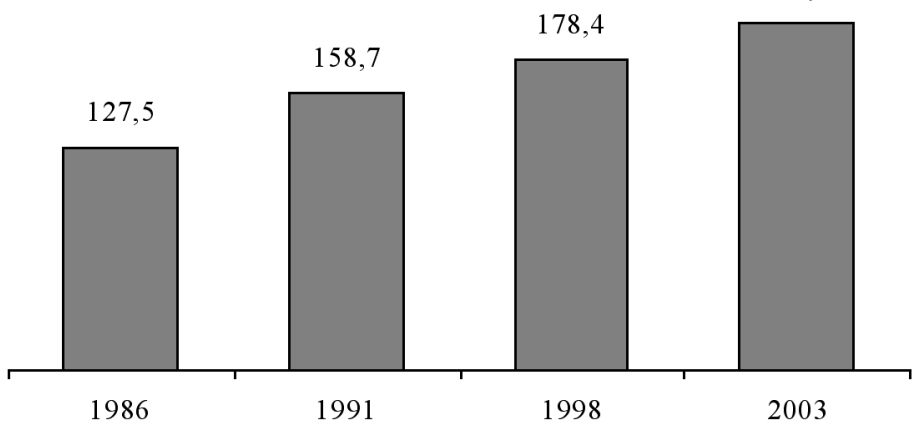

Gráfica 7. Consumo de energía en los hogares, k ep/persona, PV, 1986-2003. Fuente: IVE, diversos años.

\section{Residuos sólidos urbanos}

Los residuos sólidos urbanos son un buen indicador de la presión ejercida sobre el medio ambiente por el consumo doméstico (dado que, muy mayoritariamente, proceden de éste). La cantidad de esta categoría de residuos ha seguido un curso creciente, puesto que ha pasado de 340 kilogramos por persona y año a más de 600 entre 1986 y 2000 (gráfica 8).

Cuatro quintas partes (aproximadamente) de esa cantidad corresponden a desechos de los hogares. Los datos correspondientes son, pues, una medida indirecta pero bastante fiable del alcance de la cultura de "usar y tirar», de la rápida obsolescencia de muchos artículos de uso doméstico y del volumen creciente de envases y embalajes de todo tipo.

Los residuos urbanos son, también, uno de los ámbitos en que la predisposición favorable a hacer algo para proteger el medio ambiente está más ampliamente difundida. En 1997, dos de cada tres personas mayores de edad afirmaban que hacían lo posible para reciclar desechos del hogar y prácticamente todo el mundo valoraba positivamente las prácticas al respecto (tabla 4).

La respuesta al problema de cómo reducir la carga contaminante derivada de los residuos urbanos ha consistido básicamente en la puesta en marcha de un sistema de recuperación y recogida selectiva, basado en la colaboración voluntaria de la población y en el reciclado posterior. Este sistema tiene dos características interesantes. En primer lugar, penaliza a las personas que participan en el mismo. El número de contenedores de residuos separados es muy inferior al de residuos mezclados (gráfica 9). Eso significa que los ciudadanos y las ciudadanas que participan voluntariamente en dicho sistema, además de preocuparse por separar los residuos (una tarea que, debido a la composición de envases y productos, es muchas veces imposible), han de recorrer distancias más largas con su carga a cuestas para depositarla en los contenedores. 


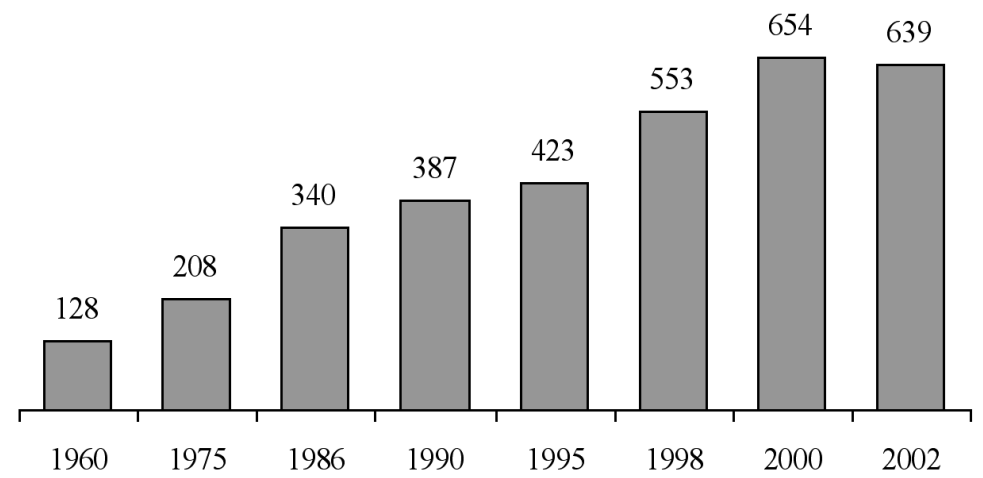

Gráfica 8. Residuos sólidos urbanos recogidos, kg por persona, PV, 1960-2002.

Fuente: INEBase (noviembre de 2005); Almenar, Bono y García, 2000.

Tabla 4. Actitudes respecto a algunas opciones de consumo.

Pregunta: ¿Cuáles de las siguientes medidas ha tomado Vd. ya?: Y cuáles piensa que podría tomar en un futuro próximo?

Población de 18 años y más, P.V., 1997, \%

\begin{tabular}{llllc}
\hline & Ya lo hago & $\begin{array}{l}\text { Podría hacerlo } \\
\text { en un futuro } \\
\text { próximo }\end{array}$ & $\begin{array}{l}\text { No lo hago } \\
\text { y tampoco } \\
\text { pienso hacerlo }\end{array}$ & Ns/Nc \\
\hline No derrochar agua corriente & 90,3 & 8,5 & 0,9 & 0,4 \\
\hline $\begin{array}{l}\text { Apagar las luces y los aparatos } \\
\text { eléctricos cuando no son } \\
\text { necesarios }\end{array}$ & 91,9 & 7,5 & 0,4 & 0,3 \\
\hline $\begin{array}{l}\text { Hacer lo que puedo para } \\
\text { reciclar residuos domésticos }\end{array}$ & 67,8 & 25,3 & 5,8 & 1,3 \\
\hline $\begin{array}{l}\text { Comer menos carne y más } \\
\text { verduras y frutas }\end{array}$ & 57,5 & 27,4 & 13,8 & 1,4 \\
\hline $\begin{array}{l}\text { Comprar bebidas con envase } \\
\text { retornable o reciclable }\end{array}$ & 43,0 & 46,0 & 6,1 & 4,9 \\
\hline $\begin{array}{l}\text { Instalar en casa un sistema } \\
\text { de energía solar }\end{array}$ & 2,6 & 36,8 & 54,0 & 6,6 \\
\hline $\begin{array}{l}\text { Usar menos el coche para } \\
\text { contaminar menos (o no } \\
\text { usarlo en absoluto) }\end{array}$ & 41,9 & 24,1 & 20,1 & 13,9 \\
\hline
\end{tabular}

Fuente: Almenar, Bono y Garcia, 2000. 


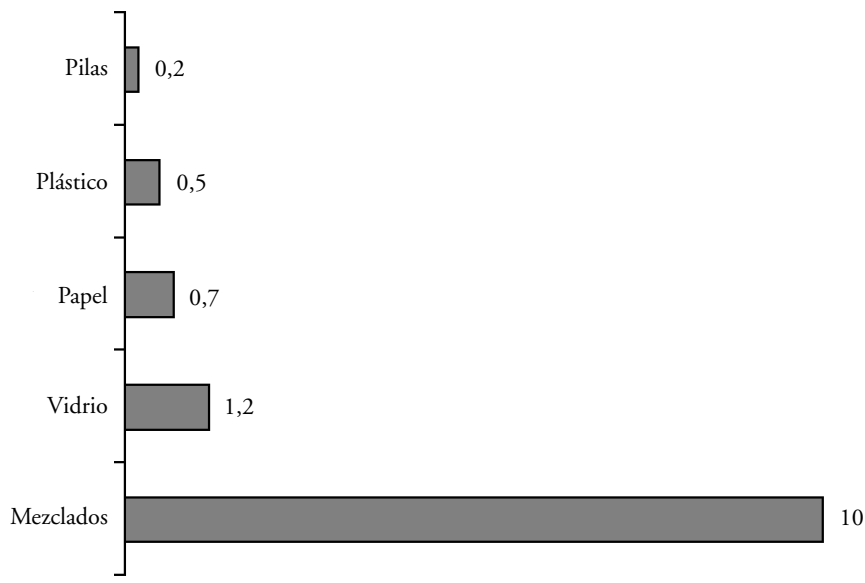

Gráfica 9. Contenedores de residuos sólidos urbanos por 1.000 habitantes, PV, 1998. Fuente: INE, 2000.

En segundo lugar, el sistema tiene unos efectos muy limitados sobre la carga total de materiales reintroducidos en el medio ambiente. En los últimos años, mediante el sistema de recogida selectiva de papel, vidrio y plásticos, se vienen recuperando unos 50 kilogramos por persona (gráfica 10). Esto significa, más o menos, que la recogida selectiva evita que vuelva al medio ambiente 1 de cada 170 kilogramos de residuos de todo tipo producidos por la sociedad valenciana (sin contar flujos ocultos). En realidad, incluso la cantidad recuperada a partir del tratamiento de residuos mezclados — sobre todo para la producción de compost- es muy superior.

La participación real de la población valenciana en la recogida selectiva es, probablemente, inferior a la disposición declarada en las encuestas. Aun así, supone millones de horas de trabajo voluntario, sin incentivos materiales (o, más exactamente, con incentivos materiales negativos), para producir un resultado que - en cuanto a su finalidad explícita (reducir la contaminación del medio ambiente) — es poco más que insignificante. La inadecuación de los sistemas de recogida selectiva y reciclaje para reducir la presión de la sociedad sobre su entorno natural ha sido ya estudiada en la sociología ecológica (Weinberg, Pellow y Schnaiberg, 2000). La explicación de que, pese a ello, el sistema consiga un grado importante de colaboración ciudadana pasa más por sus funciones latentes que por sus objetivos declarados: la recogida selectiva parece ser sobre todo un ritual de cohesión social, que permite a una parte de la población aliviar el sentimiento de culpa haciendo algo por el medio ambiente, así como sentirse parte de una tarea colectiva (sabiendo que muchas otras personas en el vecindario contribuyen también a realizarla). Por otro lado, permite a los gobiernos locales dar la impresión de que hacen algo para resolver un 


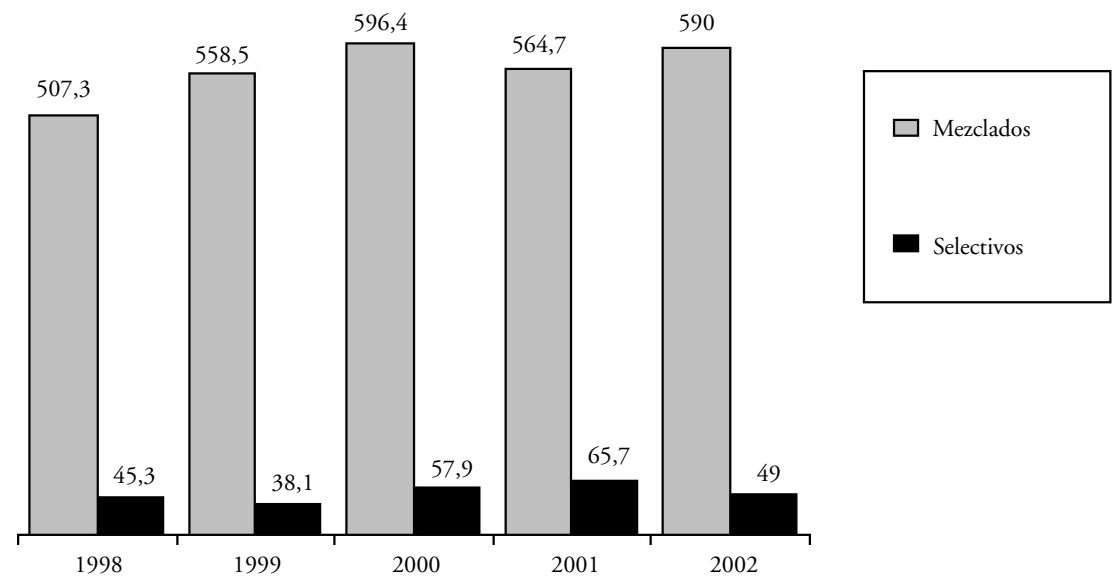

Gráfica 10. Residuos sólidos urbanos recogidos, mezclados y de recogida selectiva, PV, 19982002, kg por persona y año.

Fuente: INEBase, noviembre de 2005. Elaboración propia.

problema, el de los residuos, cada día más intratable, cada día más fuera del alcance de sus capacidades de gestión.

\section{Discusión}

El principal ámbito de actuación que, con vistas a cuidar el medio ambiente, la población en general percibe a su alcance es el consumo, mucho más que la intervención política o la intervención en los procesos productivos. Esta predisposición, sin embargo, se ve minimizada en sus efectos, porque tiene como referente un marco social con muchas características de signo contrario. Los rasgos fundamentales del modelo de consumo de masas que contribuyen a su impacto ambiental (concentración de la producción y participación creciente en la distribución de las grandes superficies, dieta descompensada — con mucha proteína y grasa animal y expansión de la comida rápida-, movilidad centrada en el coche privado, suburbanización, alta generación de residuos) aparecen consolidados y, en muchos aspectos relevantes, todavía en expansión. En formas diferentes, la difusión de nuevas prácticas de "vida moderna» a todas las capas sociales y los consumos posicionales suman sus efectos en dicho sentido.

En sentido contrario van ganando presencia las actitudes que apuntan a un cambio de los comportamientos en algunos ámbitos del consumo más cotidiano (ahorro de agua y energía, contribución al reciclaje de los residuos, presencia en la dieta de productos de la agricultura y la ganadería ecológicas, etc.). De forma convergente, viene produciéndose una expansión paulatina y mode- 
rada de los nichos de mercado para "productos verdes» en diferentes ámbitos del consumo. Este fenómeno es mucho más visible en los productos perecederos y en las pequeñas manufacturas (alimentos, productos de limpieza y aseo, algunos electrodomésticos) que en los equipamientos básicos más duraderos (construcción de la vivienda, sistemas de suministro de energía a los hogares, medios de transporte). Por ejemplo, un ámbito que, teóricamente, contiene un gran potencial para el cambio hacia la sostenibilidad, el de las infraestructuras (en particular, la vivienda), se ha mostrado hasta hoy insensible a los síntomas de cambio mencionados: la fortísima actividad constructora de la última década, ajena casi sin excepciones a cualquier consideración medioambiental, puede haber condicionado el futuro muy severamente. Este sesgo limita mucho la capacidad de las novedades aludidas para reducir sensiblemente el impacto ambiental del consumo. Incluso en ese ámbito limitado, su alcance dependerá mucho de las formas que adopten el marco legal y la iniciativa institucional (leyes de envases y embalajes, sistemas de gestión de residuos, incentivos al ahorro de recursos...), así como del grado de penetración de los "productos verdes» en las grandes estructuras de distribución (pues no parece previsible una pronta inversión de la tendencia a la concentración en las redes de distribución).

En definitiva, aunque el campo del consumo parece el más claro candidato a registrar el surgimiento y difusión de comportamientos sociales inspirados por la sostenibilidad (y, en particular, por la conexión entre sostenibilidad y salud), tanto los efectos actuales de esos comportamientos como sus posibilidades de incidencia en el futuro inmediato parecen bastante limitados. Es claro, al menos, que no han contrarrestado hasta hoy las fuerzas que empujan en dirección a una mayor presión ambiental derivada del consumo doméstico.

En buena medida, esas fuerzas radican en el sistema de producción y en las estructuras comerciales (dos puntos no tocados en esta investigación). En buena medida, pero no totalmente. Desde el «lado del consumidor» hay rasgos que apuntan en la misma dirección. El primero de ellos es la concentración de las actitudes favorables a la protección medioambiental en comportamientos que corresponden a lo que podríamos llamar el "pequeño consumo» (ahorro doméstico de agua y electricidad, envases, contribución al reciclaje de residuos, etc.), siendo sensiblemente menor el grado de acuerdo en lo relativo a decisiones que tendrían un efecto mucho mayor (cambio a energías renovables, desmotorización de la movilidad, introducción de criterios de sostenibilidad en la construcción, etc.) (tabla 4). En consecuencia, incluso si la difusión efectiva de esos comportamientos llegara a ser mayor de lo que es, su capacidad para invertir la tendencia hacia más presión ambiental sería limitada.

En cualquier caso, los efectos de esas prácticas para las que hay actitudes ampliamente favorables son todavía inapreciables. Los datos de encuesta mencionados en la tabla 4 fueron obtenidos en 1997. En torno a ese año de referencia, la evolución de los consumos de agua y electricidad en los hogares, así como de la generación de basuras, se vio poco afectada. El consumo de agua pasó de 144 litros por persona y día en 1995 a 164 en 1999; la acumulación de 
basura, de 423 kilogramos a 585; el consumo por persona de electricidad en los hogares creció un $11 \%$ de 1995 a 1998.

Un segundo rasgo que refuerza la dinámica de presión ambiental creciente es la combinación de efectos de distinción y de democratización en las modalidades de consumo más modernas. Hasta ahora, no he considerado el hecho de que determinados consumos constituyen «signos de distinción», señalan las diferencias entre los distintos grupos de estatus. Quien más tiene, más gasta, claro está, pero no más en todo. Una posición social relativamente privilegiada se manifiesta sobre todo de ciertas formas: haciéndose servir por otras personas, exhibiendo vestidos y adornos vistosos, teniendo grandes casas con artefactos sorprendentes y sofisticados, moviéndose en carrozas lujosas, demostrando que se conoce lo que otros ignoran... Los consumos posicionales o de distinción suelen ser una buena guía para examinar las tendencias generales (en épocas de expansión económica, al menos): a medida que la capacidad de gasto aumenta, los grupos sociales y los países relativamente menos favorecidos tienden a imitar los comportamientos observados en los grupos y países más favorecidos. La larga tradición sociológica de análisis y discusión de este asunto (Veblen, 1974; Bourdieu, 1988) se está ahora aplicando para comprender la difusión de los estilos de consumo en el contexto de la sociología ecológica (Wilk, 1998). Muchos de los consumos posicionales (aunque no todos) están asociados a un impacto ambiental relativamente elevado. Es el caso, por ejemplo, de la carne (en particular de las carnes rojas) y de los alimentos exóticos en la dieta. Del coche en el ámbito de la movilidad. De la casa separada del fragor urbano en la forma de alojamiento. De la rápida respuesta al cambio de la moda en cuanto al consumo de fibras. Del papel por lo que respecta a la distinción cultural. Del ritmo más rápido en la sustitución de equipamientos domésticos y productos de todo tipo en cuanto al volumen de residuos. Como consecuencia, la difusión de los estilos de consumo más modernos tiende a menudo a incrementar la presión sobre el medio ambiente.

El consumo de productos ambientalmente más costosos es a menudo más alto entre los grupos sociales con mayores ingresos (primer cuartil de la tabla 5), con niveles de educación más altos y más urbanos (García y Duart, 1998; Almenar, Bono y Garcia, 2000: 429-438). Estos grupos, por otra parte, suelen ser los primeros en acceder a las novedades aparecidas en los mercados y son la referencia a imitar por otros estratos sociales, convirtiéndose así en difusores de los requerimientos ambientales en aumento. En el periodo más reciente, algunos segmentos de esos grupos más insertos en la modernidad son también la clientela preferente de los nuevos mercados de "productos verdes». Este hecho ha dado pie a especular con la posibilidad de que el sesgo posicional ayude también a la ulterior difusión de tales productos y los estilos de vida a ellos asociados. Sin embargo, la novedad del fenómeno y su limitado alcance dificultan por el momento evaluar sus efectos.

Los dos principales terrenos de prueba del diagnóstico arriba resumido son, hoy por hoy, la alimentación y el tráfico urbano. Hay una fuerte conexión entre la alimentación y la salud, con síntomas de crisis activados por las reite- 
Tabla 5. Indicadores de presión ambiental del consumo doméstico, por cuartiles de ingresos.

PV, 1991, por persona y año (en pesetas o porcentajes)

\begin{tabular}{|c|c|c|c|c|}
\hline & $4^{0}$ cuartil & 3er cuartil & $2^{\circ}$ cuartil & 1er cuartil \\
\hline Gas ciudad y gas natural & 193 & 155 & 216 & 653 \\
\hline $\begin{array}{l}\text { Aparatos de calefacción } \\
\text { y electrodomésticos }\end{array}$ & 6.482 & 5.694 & 7.042 & 9.074 \\
\hline Automóviles & 11.445 & 13.642 & 31.185 & 51.415 \\
\hline Carburantes para transporte privado & 12.355 & 22.745 & 27.099 & 33.670 \\
\hline
\end{tabular}

Proporción del gasto en carnes

y pescados sobre el total del gasto

en alimentos

$40,08 \% \quad 41,14 \%$

$41,40 \% \quad 44,00 \%$

Proporción del gasto en alimentos

muy procesados sobre el total

del gasto en alimentos

$16,56 \%$

$18,90 \%$

$18,79 \%$

$19,95 \%$

Proporción del gasto en compra

y uso de automóviles sobre el total

del gasto

$6,45 \%$

$8,64 \%$

$11,95 \%$

$12,77 \%$

Fuente: Almenar, Bono y Garcia, 2000.

radas alarmas en este campo (desde el aceite de colza hasta las vacas locas y la gripe del pollo). Que la cuestión evolucione en un sentido favorable o adverso a la sostenibilidad dependerá de que los productos de la agricultura ecológica encuentren o no su camino hacia una población que se muestra crecientemente dispuesta a hacerlos suyos. El escenario al respecto es sumamente incierto. Algo parecido puede decirse del transporte: aunque no es de esperar que, en el inmediato futuro, el predominio del coche privado sea sustancialmente desafiado, sí son previsibles tensiones en el punto en que la crisis del modelo vigente de movilidad es más aguda: los desplazamientos urbanos (un campo en que los efectos de saturación y congestión son ampliamente reconocidos como intratables).

\section{Referencias bibliográficas y fuentes estadísticas}

Almenar, R.; Bono, E.; GarCiA, E. (dir.) (2000). La sostenibilidad del desarrollo: El caso valenciano. $2 \mathrm{a}$ ed. Valencia: Universitat de València/Fundació Bancaixa.

- (2001). Prospectiva sobre la sostenibilidad medioambiental valenciana. Valencia: Fundació Bancaixa, 3 vols., investigación no publicada.

Almenar, R.; Bono, E.; CAstelló, R.; Diago, M.; GarCia, E. (2002). 2002: La situació del País Valencià: Tendències $i$ indicadors de desenvolupament humà $i$ sostenibilitat mediambiental. Alzira: Editorial Germania/Confederació Sindical de CC.OO. del PV. 
BÖGE, S. (1996). «Freight transport, food production and consumption in the United States and in Europe, or how far can you ship a bunch of onions in the United States?». Wuppertal Papers, no 56, Wuppertal Institut für Klima, Umwelt, Energie.

BOURDIEU, P. (1988). La distinción. Madrid, Taurus.

BROWER, M.; LEON, W. (1999). The consumer's guide to effective environmental choices: Practical advice from the Union of Concerned Scientists. Nueva York: Three Rivers Press.

Cabrer, B. y otros (1999). Series económicas de la Comunidad Valenciana. Valencia: Generalitat Valenciana/Conselleria de Presidència.

Camagni, R.; Gibelli, M. C.; Rigamonti, P. (2002). «Urban mobility and urban form: the social and environmental costs of different patterns of urban expansion». Ecological Economics, 40, p. 199-216.

CARlsSON-KanYAMA, A. (1997). "Weighted average source points and distances for consumption origin: Tools for environmental impact analysis». Ecological Economics, vol. 23, p. 15-23.

- (1998). "Food consumption patterns and their influence on climate change». Ambio, vol. 27, no 7, p. 528-534.

CEH (1985). Anuari Estadistic: Comunitat Valenciana, 1984. Valencia: Generalitat Valenciana/Conselleria d'Economia i Hisenda.

- (1986). Anuari estadistic: Comunitat Valenciana, 1985. Valencia: Generalitat Valenciana/Conselleria d'Economia i Hisenda.

- (1987). Anuari estadistic: Comunitat Valenciana, 1986. Valencia: Generalitat Valenciana/Conselleria d'Economia i Hisenda.

COleY, D.A.; GoOdlife, E.; MACDIARMID, J. (1998). «The embodied energy of food: The role of diet». Energy Policy, vol. 26, no 6, p. 455-459.

COPUT (1985). Libro Blanco del agua en la Comunidad Valenciana. Valencia: Generalitat Valenciana/Conselleria d'Obres Públiques, Urbanisme i Transport.

CORES (2001). Boletín Estadístico de Hidrocarburos: Resumen año 2000. Madrid: Ministerio de Economía/Corporación de Reservas Estratégicas de Productos Petrolíferos, http://www.cores.es/boletines.html.

CROCKer, D.A.; LINDEN, T. (eds.) (1997). Ethics of consumption: The good life, justi$c e$, and global stewardship. Lanham (MD): Rowman \& Littlefield Publishers.

DuRNING, A.T. (1992). How much is enough? The consumer society and the future of the earth. Londres: Earthscan.

- (1996). The car and the city: 24 steps to safe streets and healthy communities. Seattle: Northwest Environment Watch.

EPSAR (1995). Memòria de gestió, 1994. Valencia: Generalitat Valenciana/Entitat Pública de Sanejament d'Aigües Residuals.

Estevan, A.; SANZ, A. (1996). Hacia la reconversión ecológica del transporte en España. Madrid: Los Libros de la Catarata.

Freund, P.; MARTIN, C. (1993). The ecology of the automobile. Montreal: Black Rose. GARCIA, E. (1995). El trampoli faustic: Ciència, mite i poder en el desenvolupament sostenible. Alzira: Germania.

- (1999). «La sostenibilidad de las ciudades y la organización social de la movilidad». Ecología Política, no 17, p. 55-68.

Garcia, E.; Duart, P. (1998). "Consumo y sustentabilidad en la Comunidad Valenciana». Revista Internacional de Sociología (RIS), tercera época, no 19-20, p. 247-278. 
Goodwin, N.R.; Ackerman, F.; Kiron, D. (eds.) (1997). The consumer society. Washington: Island Press.

HARLAND, E. (1995). Eco-renovation: The ecological home improvement guide. Foxhole (UK): Green Books.

HELleR, M.C.; KeOleIAN, G.A. (2000). Life-cycle-based sustainability indicators for assessment of the U.S. food system. University of Michigan: Center for Sustainable Systems, Report no CSS00-04.

IMPIVA (2000). Balance energético de la Comunidad Valenciana, 1998. Valencia: Institut de la Mitjana i Petita Empresa Valenciana/Generalitat Valenciana.

INE (1983a). Encuesta de Presupuestos Familiares 1980-1981. Tomo I: El gasto y el ingreso de los hogares: Conjunto nacional. Madrid: Instituto Nacional de Estadística.

- (1983b). Encuesta de Presupuestos Familiares 1980-1981. Tomo II: Equipamiento y condiciones de las viviendas familiares (conjunto nacional de hogares). Madrid: Instituto Nacional de Estadística.

- (1984). Encuesta de Presupuestos Familiares 1980-81. Tomo III: El consumo de alimentos, bebidas y tabaco en cantidades físicas. Madrid: Instituto Nacional de Estadística.

- (1994). Encuesta de Presupuestos Familiares 1990-91. Volumen 2: Consumo de alimentos, bebidas y tabaco en unidades fisicas. Madrid: Instituto Nacional de Estadística.

- (1995). Encuesta de Presupuestos Familiares 1990-91. Tomo IV: Resultados por CC.AA.: Comunidad Valenciana. Madrid: Instituto Nacional de Estadística.

- (1998). Encuesta Continua de Presupuestos Familiares (Renovada en el $2^{\circ}$ trimestre de 1997): Metodología. Madrid: INE, 1998.

- (2000). Estadisticas de medio ambiente: Encuesta sobre recogida y tratamiento de residuos sólidos urbanos: Año 1998. Madrid: Instituto Nacional de Estadística.

- (2001a). Estadísticas de medio ambiente: Estadísticas de residuos: Año 1999. Madrid: Instituto Nacional de Estadística.

- (2001b). Estadísticas de medio ambiente: Estadísticas del agua: Año 1999. Madrid: Instituto Nacional de Estadística.

IVE (1991). Anuari Estadistic. Comunitat Valenciana, 1990. Valencia: Generalitat Valenciana, Institut Valencià d'Estadística.

- (1993). Anuari Estadistic. Comunitat Valenciana, 1992-93. Valencia: Generalitat Valenciana, Institut Valencià d'Estadística.

- (1997). Anuari Estadistic. Comunitat Valenciana, 1997. Valencia: Generalitat Valenciana, Institut Valencià d'Estadística.

- (2000a). Anuari Estadistic. Comunitat Valenciana, 1999. Valencia: Generalitat Valenciana/Institut Valencià d'Estadística.

- (2000b). Anuari Estadistic. Comunitat Valenciana, 2000. Valencia: Generalitat Valenciana, Institut Valencià d'Estadística.

- (2002). La Comunidad Valenciana en cifras 2001. Valencia: Generalitat Valenciana/Institut Valencià d'Estadística.

LAPPÉ, F.M. (1991). Diet for a small planet. San Francisco: Food First.

LOREK, S.; SPANGENBERG, J.H. (2001). «Environmentally sustainable household consumption: From aggregate environmental pressures to indicators for priority fields of action». Wuppertal Papers no 117, Wuppertal Institut für Klima, Umwelt, Energie.

MATTHEWs, E.; Hammond, A. (1999). Critical consumption trends and implications: Degrading earth's ecosystems. Washington: World Resources Institute. 
Ministerio de Agricultura, Pesca y Alimentación (2000). La alimentación en España, 1999. Madrid: Ministerio de Agricultura, Pesca y Alimentación/Secretaría General de Agricultura y Alimentación/Dirección General de Alimentación.

- (2001). La alimentación en España, 2000. Madrid: Ministerio de Agricultura, Pesca y Alimentación/Secretaría General de Agricultura y Alimentación/Dirección General de Alimentación.

MIFO (MINISTERIO DE FOMENTO) (1998). Indice de precios de las viviendas: Estadística del precio medio del $\mathrm{m}^{2}$. Datos obtenidos de las tasaciones hipotecarias, 1997. Madrid: Ministerio de Fomento/Dirección General de Programación Económica.

- (1999). Indice de precios de las viviendas: Estadistica del precio medio del $\mathrm{m}^{2}$. Datos obtenidos de las tasaciones hipotecarias, 1998. Series estadisticas. Madrid: Ministerio de Fomento/Dirección General de Programación Económica.

- (2000). Encuesta de movilidad de las personas residentes en España. MOVILIA; $<$ http://www.mfom.es>.

- (2001a). Anuario Estadístico 2000. Madrid: Ministerio de Fomento/Dirección General de Programación Económica.

- (2001b). Atlas estadístico de la edificación de viviendas y sus precios en España: Año 2000. Madrid: Ministerio de Fomento/Dirección General de Programación Económica.

MOPT (1990). Anuario estadístico 1990. Madrid: Ministerio de Obras Públicas y Transporte.

- (1991). Anuario estadístico 1991. Madrid: Ministerio de Obras Públicas y Transporte. MOPU (1987). Anuario estadístico 1987. Madrid: Ministerio de Obras Públicas y Urbanismo.

Newman, P.; Kenworthy, J. (1999). Sustainability and cities: Overcoming automobile dependence. Washington: Island Press.

OECD (1997). Sustainable consumption and production: Clarifying the concepts. París: Organisation for Economic Co-operation and Development.

- (2002). Towards sustainable household consumption? Trends and policies in OECD countries. París: Organisation for Economic Co-operation and Development.

Pimentel, D.; Pimentel, M. (1979). Food, energy and society. Londres: Edward Arnold.

PirOG, R.; VAN PELT, T.; ENSHAYAN, K.; COOK, E. (2001). Food, fuel, and freeways: An Iowa perspective on how far food travels, fuel usage, and greenhouse gas emissions. Ames: Iowa State University, Leopold Center for Sustainable Agriculture.

Rifkin, J. (1992). Beyond beef: The rise and fall of cattle culture. Nueva York: Dutton. Ritzer, G. (1996). The McDonaldization of society. Revised edition. Thousand Oaks (CA): Pine Forge Press.

Rosenblatt, R. (ed.) (1999). Consuming desires: Consumption, culture, and the pursuit of happiness. Washington: Island Press.

Ryan, J.; DuRning, A. (1997). Stuff: The secret lives of everyday things. Seattle: Northwest Environment Watch.

SACHS, W. (1992). For love of the automobile: Looking back into the history of our desires. Berkeley: University of California Press.

SACHS, W.; LOSKE, R.; LiNZ, M. y otros (1998). Greening the North: A post-industrial blueprint for ecology and equity. Londres: Zed Books.

SCHLOSSER, E. (2001). Fast food nation: What the all-american meal is doing to the world. Londres: Penguin.

SMIL, V. (2001). Feeding the world: A challenge for the twenty-first century. Cambridge (MA): The MIT Press. 
Stanners, D.; Bourdeau, P. (eds.) (1995). Europe's environment: The Dobris assessment. Copenhague: European Environment Agency.

Stern, P.C.; DieTZ, T.; RuTTAN, V.W.; SOCOlow, R.H.; SwEENEY, J.L. (eds.) (1997). Environmentally significant consumption: Research directions. Washington: National Academy Press.

TansY, G.; D’SiLva, J. (eds.) (1999). The meat business: Devouring a hungry planet. Londres: Earthscan.

Teitel, M. (1992). Rainforest in your kitchen: The hidden connection between extinction and your supermarket. Washington: Island Press.

UNDP (United Nations Development Programme) (1998). Human development report 1998: Consumption for human development. Nueva York: Oxford University Press.

Veblen, T. (1974) [1899]. Teoría de la clase ociosa. México: Fondo de Cultura Económica.

WeinberG, A.S.; Pellow, D.N.; Schnaiberg, A. (2000). Urban recycling and the search for sustainable community development. Princeton (NJ): Princeton University Press.

WILK, R. (1998). «Emulation, imitation, and global consumerism». Organization and Environment, vol. 11, no 3, p. 314-333.

WRI (2001). World Resources 2000-2001. Washington: World Resources Institute. 